\title{
Orthopedic Surgery and Telemedicine in Times of COVID-19 and Beyond: a Review
}

\author{
Philippe Moisan $^{1}$ (D) $\cdot$ Bardia Barimani $^{1} \cdot$ John $^{\text {Antoniou }}{ }^{2}$ \\ Accepted: 8 January 2021 / Published online: 18 January 2021 \\ (C) The Author(s), under exclusive licence to Springer Science+Business Media, LLC part of Springer Nature 2021
}

\begin{abstract}
Purpose of Review This review article presents the current knowledge on the use of telemedicine and summarizes the literature highlighting the advantages and limitations of this technology in the field of orthopedic surgery during the COVID-19 pandemic and beyond.

Recent Findings Orthopedic surgery is the surgical specialty that has seen the highest proportion of its procedures cancelled due to the pandemic. In this context and onward, telemedicine seems to be a reasonable option for the orthopedic surgeon. Multiple studies have described its safety and a similar patient satisfaction compared to in-person consultations. It has a potential to increase productivity and decrease wait times by providing easier access to the clinician and by decreasing travel-associated limitations and costs. Authors have described the possibility to conduct a reliable virtual assessment of the patient range of motion. Some of the limitations to the use of this technology are technological literacy and access to virtual consultation platforms, the inability to conduct a complete physical examination, potential reduction in identification of intimate and child abuse victims, and limited knowledge about the legal implications of this technology.

Summary Telemedicine in orthopedic surgery has a potential to increase productivity, reduce costs, and increase the access to healthcare. Identified limitations include risk of misdiagnosis, required technologic literacy, unknown legal implications, and failure to identify victims of abuse. In order to use this technology judiciously, the clinician must take into consideration the patient's condition and his technological literacy and be aware of the advantages and disadvantages.
\end{abstract}

Keywords Telemedicine $\cdot$ Teleorthopedics $\cdot$ COVID-19 $\cdot$ Virtual consultations $\cdot$ Virtual physical examination $\cdot$ Orthopedic surgery

\section{Introduction}

Traditionally, the practice of medicine has involved in-person consultations and examinations. In a period where physical proximity puts the patient and the doctor at increased risk of viral transmission and where orthopedic care is limited to urgent or

Philippe Moisan

philippe.moisan@mail.mcgill.ca

Bardia Barimani

bardia.barimani@mail.mcgill.ca

1 Division of Orthopaedic Surgery, McGill University Health Centre, 1650 Cedar Avenue, Montreal, Quebec H3G 1A4, Canada

2 Division of Orthopaedic Surgery, McGill University Health Centre, Jewish General Hospital, Montreal, Quebec, Canada emergency visit [1], physicians are forced to rethink the paradigms on which they have based their practice for many years.

The COVID-19 pandemic has hastened the process of shifting towards telemedicine [2]. Teleorthopedics involves the delivery of care between a patient and an orthopedic surgeon remotely. Commonly used technology platforms are laptops or computers with a webcam and a tablet or a phone with an integrated camera. Applications such as Microsoft Teams and Zoom are popular and commonly used software that facilitate video consultations and the management of patient. This technology is continuously developing more so now than ever to maintain continuity of care at a time when physical contact is discouraged.

This review article aims to present the impacts of the COVID-19 pandemic on the field of orthopedic surgery, suggests evidence-based ways that surgeons can use telemedicine to their advantage, and highlights limitations and its impact on the future of orthopedic surgery. 


\section{Impact of COVID-19 on the Practice of Orthopedic Surgery}

The COVID-19 pandemic has had a considerable impact on the practice of orthopedic surgery around the world. It has led to widespread cancellation of elective surgery, delays in treatment, and putting the patient and staff at risk of contracting COVID-19. National Health Service (NHS) hospitals in England have suspended all non-urgent elective surgery for at least 3 months from April 15, 2020 ${ }^{3}$. The USA and Canada as well as many European and Asian countries have opted for a similar strategy. Consequently, orthopedic surgery resident training has been affected due to the reduced number of operative cases, clinics, and teaching received, leading to questions as to whether residency training time will be extended to make up for this loss. [3••]

Globally, more than 28 million surgeries were cancelled or postponed during the peak 12 weeks of the COVID-19 pandemic [4•], between March and May 2020. Nepogodiev et al. estimated a median number of 244,121 orthopedic surgeries cancelled in the peak 12 weeks of the pandemic in Canada, making orthopedic surgery the specialty with the highest percentage of surgical cases to be cancelled or postponed [4]. In the absence of an effective treatment or mass SARS-CoV-2 immunization, reductions in orthopedic interventions will likely continue for many more months to come.

Following this pandemic, the delays to obtain surgical treatment are expected to increase significantly and clearing this backlog will take a significant amount of time. Assuming a 20\% increase in baseline surgical volume, clearing the back$\log$ caused by the cancellations during the peak 12 weeks of the pandemic would take a median of 45 weeks. At least 90 weeks will be required if only $10 \%$ increase in volume is possible $[4 \bullet \bullet]$.

Delays to treatment are not only caused by surgery cancellations due to emergency hospital protocols, but also there are multiple reports of patients failing to seek urgent medical care due to the fear of contracting the virus [5•]. This fear by patients is in fact justified as in the absence of an effective vaccine against SARS-CoV-2, research has indicated that surgical patients are at a high risk of being exposed to the virus in the perioperative period ${ }^{4}$. Clinical characteristics and early prognosis of COVID-19 in patients with fractures appear to be more severe than patients without fractures ${ }^{67}$ which would suggest earlier presentation outweighs the risk of contracting the virus in certain circumstances. Additionally, orthopedic surgeons appear to be at a significant risk of contracting COVID-19 in the hospital setting as a survey performed in Wuhan, China, saw between 1.5 and $20.7 \%$ of orthopedic surgeons contract COVID-19 from 8 hospitals in less than 1 month $^{8}$. [6•] [7].

It is known that many surgical pathologies have worse outcomes with delayed treatment leading to worse outcomes and an increased patient mortality [8].[4•] The effects of
COVID-19 will undeniably be felt by the orthopedic community and its patients for many years to come. A key method that has been used to negate the lack of follow-up and the inability to do in-person consultations is by using telemedicine. This technology has significant advantages but also limitations and risks that should not be neglected.

Education and teaching is an important aspect of an academic orthopedic surgeon's practice. Resident education has been hindered by the reduced operating room time, patients in clinics, and one-on-one teaching by surgeons. Virtual teaching environments via videoconferencing have been used successfully for teaching trainees and educating patients and for telerehabilitation [9]. Remote learning is thought to be as effective as the traditional instructor-led methods [10]. This learning method allows the acquisition of theoretical knowledge but does not allow residents and medical learners to acquire technical and surgical skills. Virtual patient cases have been studied as an option to replace some patient encounters and they have shown modest improvement in knowledge and seem to be useful to prepare or to reinforce in-person patient encounters [11].

\section{Advantages of Teleorthopedics}

It has been reported that teleorthopedics in an outpatient setting is safe and without serious adverse events [12]. Teleconsultation services were originally introduced to reduce cost, improve efficiency, and increase quality and access to healthcare services [13]. During the COVID-19 pandemic, the main motivation for its use has been to provide continuity of care while maintaining physical distancing.

Teleorthopedics has been successful in maintaining physical distancing between patients and orthopedic surgeons. By comparing the cost of implementing and running a teleconsultation service to the cost savings associated with the patient not having to travel and miss work to consult a physician, The break-even point has been achieved after 151 teleconsultations per year [14*0]. A research group found that first-time patient visits to a tertiary orthopedic oncology center saw a reduction in costs between 12.2 and $72 \%$ when telemedicine was used for the consultation [15]. Overall, patients seem to be similarly satisfied with a teleconsultation than with an in-person consultation [16] and follow-up rates increase when barriers to accessibility such as travel distance and associated costs are eliminated [17].

Physical examination is an essential component of the orthopedic assessment. Teleconsultations do not allow the clinician to examine the patient. However, studies have evaluated the precision and reliability of a virtual physical examination and this is done by having the patient go through a series of range of motion and by assessing mobility with a virtual goniometer. [18] Dent et al. studied 52 healthy control patients 
who underwent elbow range-of-motion measurements in person, through teleconference, and with still photography. The authors reported high inter-method reliability between teleconference-based goniometry and in-person measurements. Similar accuracy and greater precision in assessing shoulder abduction and internal rotation, elbow flexion, hip abduction, and knee extension have been documented with virtual goniometry compared to visual assessment [18, 19]. The future of virtual physical examination and telerehabilitation is promising and will likely incorporate augmented reality, artificial intelligence, and sensor-based technologies with success of this method already being documented by a group in Denmark already using this to guide rehabilitation following knee arthroplasty ${ }^{15}$.

\section{Limitations of Telemedicine}

Telemedicine has many good aspects with regard to increasing efficiency and reducing costs, but limitations have been highlighted with the lack of in-person physical examinations, barriers to accessibility, and the validity of the virtual examinations, especially in acute and complex conditions. According to an online survey of 781 patients that experienced telemedicine, the main disadvantages are the lack of hands-on care, the lack of intimacy, and technical difficulties [20]. The main goal of telemedicine is to improve accessibility of professional expertise to the patient. The opposite effect was observed when trying to implement such a system with the elderly population due to their limited access to the equipment or technical knowledge necessary to participate in teleconsultations [21]. For this reason, a lot of surgeons opted to use standard telephone consultation to alleviate this issue [22]. In-person physical communication and examinations are an important part of the consultation as it can directly express care, compassion, and comfort [23]. Teleorthopedics does not allow this type of interaction which might impact the doctorpatient relationship and lead to sub-optimal care.

Studies on the virtual physical examination have demonstrated the ability to assess the range of motion in healthy adults. With the current technology, it is not possible to remotely assess the temperature, to palpate, or to physically stress and test joints. In that sense, the virtual physical examination provides very limited data and an incomplete clinical picture. Evidence to indicate the role of teleconsultation in an acute presentation or complication does not exist.

Studies evaluating patient satisfaction with telemedicine might underestimate the role that in-person consultations have in breaking social isolation and identifying child, elder, or domestic abuse and violence [24]. In fact, one in six female patients presenting to an orthopedic fracture clinic is a victim of domestic abuse and almost $2 \%$ are presenting as a direct consequence of physical abuse [25]. Orthopedic surgeons have an important role and opportunity in identifying these cases. Telemedicine limits the ability to take a clear history and does not always provide the setting necessary for such disclosures or observation of interactions with their partner.

\section{Future of Teleorthopedics}

The COVID-19 pandemic has forced the field of orthopedic surgery to adapt quickly and has hastened the implementation of telemedicine. Knowledge of key benefits and limitations of teleorthopedics will allow orthopedic surgeons to optimize the use of this technology to improve productivity, lower waiting times, and reduce costs while protecting the patient and surgeon's safety. In order to do so, clear indications for the use of teleorthopedics instead of in-person consultation need to be defined.

In some scenarios, teleconsultations might be sufficient when combined with the review of a patient's recent radiological studies. As an example, during a follow-up for a simple fracture managed conservatively, the clinician might feel comfortable doing a videoconference assessment given that he is satisfied with the radiological images and reassured by the condition of the patient. Other situations in which patients might benefit safely from teleorthopedics are long-term arthroplasty follow-ups, non-urgent referrals from general practitioners, and in patients being treated conservatively.

A conservative recommendation would be to consider inperson contact when a component of acuity, when a risk of severe complications, or when a teleconsultation does not provide sufficient clinical information for safe decision-making. It remains the responsibility of the clinician to weigh the risk of in-person contact and possible patient absenteeism compared to the possibility of an incomplete clinical assessment. The level of experience and comfort of the clinician and the patient in regard to telemedicine needs to be taken into account.

\section{Discussion}

Current indications for the use of teleconsultations in an orthopedic setting are yet to be established. The diagnostic value of virtual examinations is currently unknown and additional challenge presents itself when the patient suffers from an acute pathology or complication. Caution needs to be exerted until further studies have been made and indications are clearly established.

Access to teleconsultation requires a level of technological literacy that often times the elderly population does not possess. Increasing the use of teleconsultation or making it the new standard might have the effect of limiting the access to care and discriminating against this population group. It is 
important that instauration of teleorthopedic programs should be coupled with technical support and alternative options.

Little knowledge exists regarding the legal implications and ramifications that come with teleconsultations. Jurisprudence in context of a virtual physical examination and remote orthopedic assessment is extremely limited. This emphasizes the importance of caution when dealing with orthopedic issues where a degree of ambiguity or risk for complications is high.

\section{Conclusion}

Widespread teleorthopedics is a new reality to which we are confronted given the current COVID-19 pandemic. Most surgical procedures and clinics have been cancelled due to the risk of transmission and to free hospital beds and maintain intensive care unit capacity. These cancellations will undeniably create an important backlog that will take multiple months or years to clear [4]. Important delays in treatment and consultations are to be expected which might lead to poor patient outcomes and significant monetary costs. Telemedicine is one of the solutions that clinicians should strongly consider continuing and perfecting after the pandemic. Many authors have described its safety, the potential for increased productivity, possible cost reduction, and similar patient satisfaction rates. Caution must nonetheless be exerted due to the limited knowledge regarding its use in acute care, the validity of a virtual physical examination, and the potential for paradoxical reduction in accessibility in older populations. Until further studies are published detailing indications and contraindications, teleorthopedics needs to be used with caution while weighing the pros and cons and considering the patient and the surgeon's preference and technological literacy.

\section{Compliance with Ethical Standards}

Conflict of Interest Philippe Moisan, Bardia Barimani, and John Antoniou declare that they have no conflict of interest.

Human and Animal Rights and Informed Consent This article does not contain any studies with human or animal subjects performed by any of the authors.

\section{References}

Papers of particular interest, published recently, have been highlighted as:

- Of importance

•• Of major importance

1. CMS. CMS adult elective surgery and procedures recommendations.
2. CMS, CM, PCG, Dpipd. Telehealth services [Internet]. 2020 [cited 2020 May 21]. Available from: https://www.cms.gov/newsroom/fact-

3.・ Iacobucci G. Covid-19: all non-urgent elective surgery is suspended for at least three months in England. BMJ. 2020 Mar $18 ; 368: \mathrm{m} 1106$. Study providing key data and statistics on the surgery cancellations due to COVID-19.

4. Nepogodiev D, Bhangu A. Elective surgery cancellations due to the COVID-19 pandemic: global predictive modelling to inform surgical recovery plans. Br J Surg [Internet]. 2020 May 12 [cited 2020 May 25]; Available from: http://doi.wiley.com/10.1002/bjs.11746. Study presenting a predictive model for the recovery of the surgical waiting lists created by COVID-19.

5. Masroor S. Collateral damage of COVID-19 pandemic: delayed medical care. J Card Surg. 2020 Study explaining the consequences of delayed medical care caused by COVID-19.

6. Mi B, Chen L, Xiong Y, Xue H, Zhou W, Liu G. Characteristics and early prognosis of COVID-19 infection in fracture patients. $\mathrm{J}$ Bone Jt Surg. 2020 May;102(9):750-8. Study demonstrating that orthopedic surgeons are at a significant risk of contracting COVID-19.

7. Guo X, Wang J, Hu D, Wu L, Gu L, Wang Y, et al. Survey of COVID-19 disease among orthopaedic surgeons in Wuhan, People's Republic of China. J Bone Jt Surg. 2020 Apr;102(10):1.

8. Shin DW, Cho J, Kim SY, Guallar E, Hwang SS, Cho B, et al. Delay to curative surgery greater than 12 weeks is associated with increased mortality in patients with colorectal and breast cancer but not lung or thyroid cancer. Ann Surg Oncol. 2013 Aug;20(8):246876.

9. Baruffaldi F, Giangiacomo L, Paltrinieri A, Toni A. Videoconferencing for distance training in orthopaedics. J Telemed Telecare. 2003;9(4):241-242. https://doi.org/10.1258/ 135763303322225607.

10. Ruiz JG, Mintzer MJ, Leipzig RM. The impact of e learning in medical education. Acad Med. 2006;81(3):207-212. https://doi. org/10.1097/00001888-200603000-00002.

11. Jeimy S, Wang JY, Richardson L. Evaluation of virtual patient cases for teaching diagnostic and management skills in internal medicine: A mixed methods study. BMC Res Notes. 2018;11(1). https://doi.org/10.1186/s13104-018-3463-x.

12. Buvik A, Bugge E, Knutsen G, Småbrekke A, Wilsgaard T. Quality of care for remote orthopaedic consultations using telemedicine: a randomised controlled trial. BMC Health Serv Res. 2016 Sep;8: 16(1).

13. Bashshur RL, Shannon G, Krupinski EA, Grigsby J. Sustaining and realizing the promise of telemedicine. Telemed e-Health. 2013 May 1;19(5):339-45.

14.• Buvik A, Bergmo TS, Bugge E, Smaabrekke A, Wilsgaard T, Olsen JA. Cost-effectiveness of telemedicine in remote orthopedic consultations: randomized controlled trial. J Med Internet Res. $2019 \mathrm{Feb} 1 ; 21(2)$. Study showing the cost-benefit of teleconsultations and establishing the cost breakpoint benefit at 150 teleconsultations per year.

15. Aponte-Tinao LA, Farfalli GL, Albergo JI, Plazzotta F, Sommer J, Luna D, et al. Face to face appointment vs. telemedicine in first time appointment orthopedic oncology patients: a cost analysis. In: Studies in health technology and informatics. IOS Press; 2019. p. 512-5.Study showing the cost-benefit of a first teleconsultation for a first orthopedic visit in a tertiary center.

16. Sathiyakumar V, Apfeld JC, Obremskey WT, Thakore RV, Sethi MK. Prospective randomized controlled trial using telemedicine for follow-ups in an orthopedic trauma population: a pilot study. $\mathrm{J}$ Orthop Trauma. 2015 Mar 26;29(3):e139-45.

17. Denino WF, Osler T, Evans EG, Forgione PM. Travel distance as factor in follow-up visit compliance in postlaparoscopic adjustable gastric banding population. Surg Obes Relat Dis. 2010 Nov;6(6): 597-600. 
18. Russo RR, Burn MB, Ismaily SK, Gerrie BJ, Han S, Alexander J, et al. Is digital photography an accurate and precise method for measuring range of motion of the hip and knee? J Exp Orthop. 2017 Dec;1:4(1).

19. Russo RR, Burn MB, Ismaily SK, Gerrie BJ, Han S, Alexander J, et al. Is digital photography an accurate and precise method for measuring range of motion of the shoulder and elbow? J Orthop Sci. 2018 Mar 1;23(2):310-5. Study showing the precision of using virtual goniometry to access range of motion virtually.

20. Spear KL, Auinger P, Simone R, Ray Dorsey E, Francis J. Patient views on telemedicine for Parkinson disease. J Parkinsons Dis. 2019;9(2):401-4.

21. McLiesh P. Telehealth in contemporary orthopaedic nursing. Vol. 33, International Journal of Orthopaedic and Trauma Nursing. Elsevier Ltd; 2019. p. 1-3.

22. Downes MJ, Mervin MC, Byrnes JM, Scuffham PA. Telephone consultations for general practice: a systematic review. Syst Rev
[Internet]. 2017 Dec 3 [cited 2020 Jun 2];6(1):128. Available from: http://systematicreviewsjournal.biomedcentral.com/articles/10. 1186/s13643-017-0529-0

23. Singh C, Leder D. Touch in the consultation. Vol. 62, British Journal of General Practice. Royal College of General Practitioners; 2012. p. 147-148.

24. Flaherty EG, Sege R. Barriers to physician identification and reporting of child abuse. Vol. 34, Pediatric Annals. Slack Incorporated; 2005. p. 349-356.

25. Petrisor BA, Drew B, Rajaratnam K, Williams D, Wong I, Kwok D, et al. Prevalence of abuse and intimate partner violence surgical evaluation (PRAISE) in orthopaedic fracture clinics: a multinational prevalence study. Lancet. 2013 Sep 7;382(9895):866-76.

Publisher's note Springer Nature remains neutral with regard to jurisdictional claims in published maps and institutional affiliations. 